Proceedings of the 2007 Winter Simulation Conference

S. G. Henderson, B. Biller, M.-H. Hsieh, J. Shortle, J. D. Tew, and R. R. Barton, eds.

\title{
Combining Network Reductions and Simulation to Estimate Network Reliability
}

\author{
Abdullah Konak \\ Information Sciences and Technology \\ Penn State Berks \\ Reading, PA 19610, U.S.A.
}

\begin{abstract}
Network reduction techniques are mainly used with exact approaches such as factoring to compute network reliability. However, exact computation of network reliability is feasible only for small sized networks. Simulation is an alternative approach to estimate network reliability. This paper discuses the effect of using network reductions before estimating network reliability using a simulation. Theoretical and empirical results are provided to understand the source of variance reduction in simulation due to network reductions.
\end{abstract}

\section{INTRODUCTION}

In the connectivity-based network reliability analysis, a network is usually modeled as a probabilistic network $G(V, E)$ where $V$ is the set of nodes and $E$ is the set of unreliable arcs such that each arc $(i, j) \in E$ can be in either operative state $\left(x_{(i, j)}=1\right)$ or failed state $\left(x_{(i, j)}=0\right)$ with respective probabilities $p_{(i, j)}$ and $1-p_{(i, j)}$. Assuming that the state of each arc is independent from the others, the probability of observing a particular network state $\mathbf{x}=\left\{x_{(i, j)}:(i, j) \in E\right\}$ is given as

$$
\operatorname{Pr}\{\mathbf{x}\}=\prod_{(i, j) \in E}\left[1-p_{(i, j)}+x_{(i, j)}\left(2 p_{(i, j)}-1\right)\right]
$$

This basic model is adequate to represent many practical scenarios in real life networks. The main objective of the connectivity-based network reliability analysis is to calculate the probability that a network $G$ provides a specified connectivity service. This probability is calculated as

$$
R(G)=E[\Phi(\mathbf{x})]=\sum_{\mathbf{x} \varepsilon S} \operatorname{Pr}\{\mathbf{x}\} \Phi(\mathbf{x})
$$

where $S$ is the state space of all network states and $\Phi(\mathbf{x})$ is a binary structure function based on the connectivity service that the network is expected to provide. For example, in all-terminal reliability (i.e., the probability that all nodes of a network are connected), the structure function is defined as

$$
\Phi(\mathbf{x})= \begin{cases}1, & \text { if all nodes are connected for } \mathbf{x} \\ 0, & \text { otherwise }\end{cases}
$$

Exact computation of (2) for a connectivity-based reliability measure is NP-hard in general networks (Ball 1979). Exact methods such as factoring (Colbourn 1987, Page and Perry 1991, Resende 1986, Resende 1988, Satyanarayana and Chang 1983) and backtracking (Ball and Slyke 1977) to compute (2) are computationally feasible only for small sized networks. Several bounding approaches have been proposed to approximate (2). However, some of these bounds (Slyke and Frank 1972) are only applicable to networks with identical arc reliabilities. Bound methods that can be used with networks with different arc reliabilities (Lomonosov and Polesskii 1972, Aboelfotoh and Colbourn 1989, Brecht and Colbourn 1988, Li and Silvester 1984, Lam and Li 1986, Yang and Kubat 1989) are not tight enough, or they require considerable computational effort to obtain a good approximation.

Because of the reasons briefly summarized above, Monte Carlo (MC) simulation has been a popular approach to evaluate (2), especially for large-sized networks. The application of simulation to estimate network reliability is outwardly straightforward. In the most basic simulation approach, called Crude Monte Carlo (CMC) simulation, $K$ independent random samples of network states $\mathbf{x}^{(1)}, \ldots, \mathbf{x}^{(K)}$ are taken from $S$ according to probability function (1), and then the unbiased estimator of $R(G)$ is calculated as

$$
\widehat{R}(G)=\frac{1}{K} \sum_{k=1}^{K} \Phi\left(\mathbf{x}^{(k)}\right)
$$

with an estimator variance of

$$
\operatorname{Var}[\widehat{R}(G)]=\frac{R(G)(1-R(G))}{K} .
$$


In CMC, a random state vector $\mathbf{x}^{(k)}=\left\{x_{(i, j)}^{k}:(i, j) \in E\right\}$ is generated by individually sampling the state of each arc $(i, j) \in E$ as follows:

$$
x_{(i, j)}^{k}= \begin{cases}1, & U \leq p_{(i, j)} \\ 0, & \text { otherwise }\end{cases}
$$

where $U$ is a random number.

Although the implementation of CMC is straightforward, it has been criticized for being inefficient, especially for highly reliable networks. Therefore, alternative sampling techniques such as dagger sampling (Kumamoto et al. 1980), stratified sampling (Slyke and Frank 1972), the Markov model (Mazumdar, Coit, and McBride 1999), the sequential construction/destruction methods (Easton and Wong 1980, Fishman 1986a), importance sampling using bounds (Fishman 1986b), the graph evolution method (Elperin, Gertsbakh, and Lomonosov 1991), sampling based on failures sets (Kumamoto, Tanaka, and Inoue 1977), and the recursive variance-reduction algorithm (Cancela and Khadiri 1995, Cancela and Khadiri 1998, Cancela and Khadiri 2003) have been proposed in the literature to improve efficiency and effectiveness of the simulation approach to estimate network reliability.

Simulation has also been widely used as the primary reliability evaluation method by researchers in the design of reliable networks (Deeter and Smith 1998, Srivaree-ratana, Konak, and Smith 2002). The variance of the reliability estimator is particulary important when the simulation output is used within an optimization algorithm to evaluate candidate networks with respect to reliability. In such cases, the noise due to estimation error may cause incorrect evaluation of solutions, impairing the performance of a search algorithm.

Reliability-preserving network reductions are mainly used with the exact methods (Page and Perry 1991, Resende 1986, Resende 1988) to calculate network reliability. This paper analyzes the reduction in the variance of a simulation estimator when a network is simplified using reliabilitypreserving network reductions. The primary contribution of this paper is to provide insights on how reliabilitypreserving network reductions can provide a significant reduction in the estimator variance. In addition, the effect of using network reductions along with a variance-reduction simulation technique such as the Sequential Construction (SC) method (Easton and Wong 1980) is demonstrated. A theoretical analysis is provided for CMC, and computational experiments are carried out to understand the relationship between variance reduction and various network features such as network density and arc reliability in the case of all-terminal reliability.

\section{NETWORK REDUCTIONS AND SIMULATION}

In reliability-preserving network reductions, the objective is to reduce a network $G$ to a simpler network $G^{\prime}$ (i.e., a network with fewer nodes and/or arcs) such that

$$
R(G)=\lambda R\left(G^{\prime}\right)
$$

where $\lambda$ is a reliability-preserving multiplicative constant that depends on the applied reduction. Network reductions are primarily used with factoring approaches in the exact calculation of reliability to improve the computational performance. Network reduction can be implemented before simulation and reliability estimation for a reduced network $G^{\prime}$ can be used to estimate the reliability of the original network as follows:

$$
\hat{R}(G)=\lambda \hat{R}\left(G^{\prime}\right)
$$

There are three basic reliability-preserving reductions that can be applied in case of all-terminal reliability.

\subsection{Degree-1 Reduction}

If network $G$ has a node $i$ with only one single incident arc $(i, j)$, then node $i$ and its incident arc $(i, j)$ are removed from the network to obtain $G^{\prime}$. In this case, $R(G)=p_{(i, j)} R\left(G^{\prime}\right)$.

\subsection{Degree-2 Reduction}

This reduction is applied to nodes that have only two incident arcs. Assume that node $i$ has only two incident arcs $(i, j)$ and $(i, k)$. To obtain $G^{\prime}$, node $i$ is removed from the network, and in addition, $\operatorname{arcs}(i, j)$ and $(i, k)$ are replaced by a new arc $(j, k)$ with $p_{(j, k)}=p_{(i, j)} p_{(i, k)} /\left(p_{(i, j)}+p_{(i, k)}-p_{(i, j)} p_{(i, k)}\right)$. In this case, $R(G)=\left(p_{(i, j)}+p_{(i, k)}-p_{(i, j)} p_{(i, k)}\right) R\left(G^{\prime}\right)$.

\subsection{Parallel Arc Reduction}

In parallel arc reduction, two parallel arcs $\left(i_{1}, j_{1}\right)$ and $\left(i_{2}, j_{2}\right)$ between nodes $i$ and $j$ are substituted by a single arc $(i, j)$ with $p_{(i, j)}=1-\left(1-p_{\left(i_{1}, j_{1}\right)}\right)\left(1-p_{\left(i_{2}, j_{2}\right)}\right)$, and $R(G)=R\left(G^{\prime}\right)$ in this case. It should be noted that even though an original network $G$ does not have any parallel arcs, they may occur as a result of degree- 2 reductions.

The proposed approach is very straightforward and can be summarized as follows: 


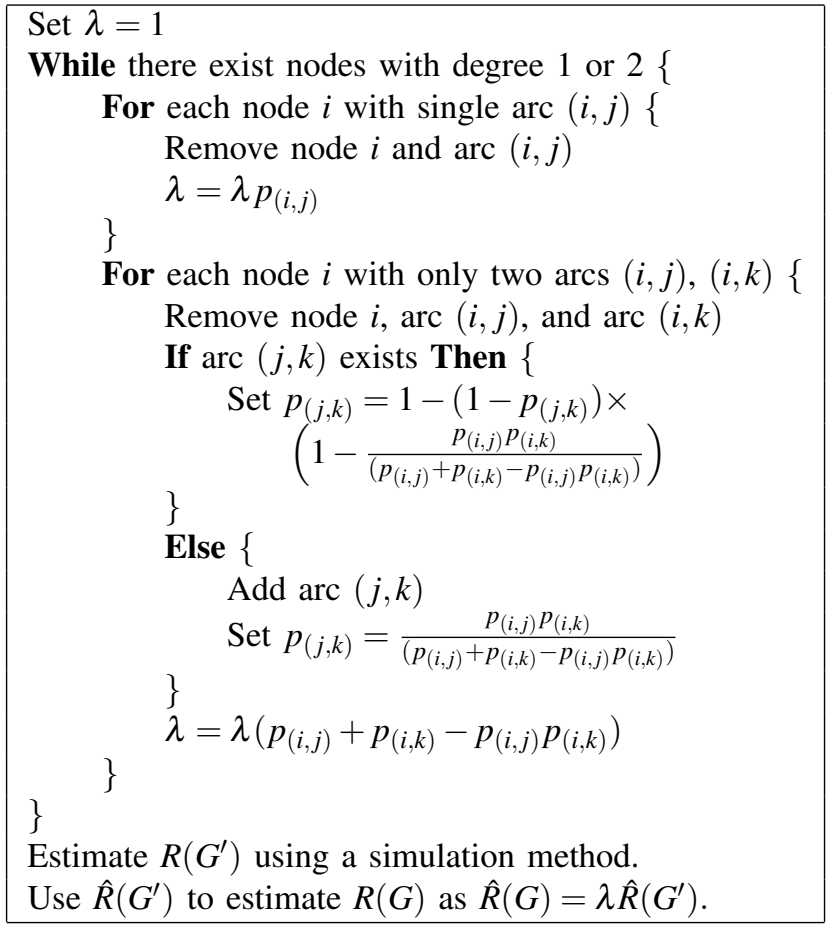

In the procedure above, the variance of estimator $\hat{R}(G)$ can be calculated from the samples taken during the simulation.

Corollary 1 In the procedure above, if CMC is used to estimate $R\left(G^{\prime}\right)$, then the variance of $\hat{R}(G)$ is given by

$$
\operatorname{Var}[\widehat{R}(G)]=\frac{R(G)(\lambda-R(G))}{K} .
$$

Proof: From (7), $R\left(G^{\prime}\right)=\frac{R(G)}{\lambda}$. Substituting this into (5), the variance of $R\left(G^{\prime}\right)$ is written as

$$
\operatorname{Var}\left[\widehat{R}\left(G^{\prime}\right)\right]=\frac{\frac{R(G)}{\lambda}\left(1-\frac{R(G)}{\lambda}\right)}{K}=\frac{R(G)(\lambda-R(G))}{\lambda^{2} K}
$$

From (8), $\operatorname{Var}[\hat{R}(G)]=\lambda^{2} \operatorname{Var}\left[\hat{R}\left(G^{\prime}\right)\right]$, and substituting (10) into this yields $\operatorname{Var}[\widehat{R}(G)]=\frac{R(G)(\lambda-R(G))}{K}$. The variance reduction factor due to the applied network reductions is given as

$$
\delta=\frac{\operatorname{Var}[] \text { before reductions }}{\operatorname{Var}[] \text { after reductions }}=\frac{1-R(G)}{\lambda-R(G)} .
$$

\section{Computational Experiments}

In this section, the reduction in an estimator variance due to network reductions is analyzed using randomly generated 10-node networks with identical arc reliabilities. In order to investigate the effect of network reductions with respect to network density (i.e., the ratio of the number of arcs to the number of nodes), networks with the number of arcs
Table 1: Results for CMC simulation.

\begin{tabular}{cccc}
\hline & \multicolumn{3}{c}{$\delta$} \\
\cline { 2 - 4 }$p$ & minimum & median & maximum \\
\hline 0.90 & 2 & 31 & 6897 \\
0.95 & 4 & 115 & 129293 \\
0.99 & 15 & 2566 & 95284433 \\
\hline
\end{tabular}

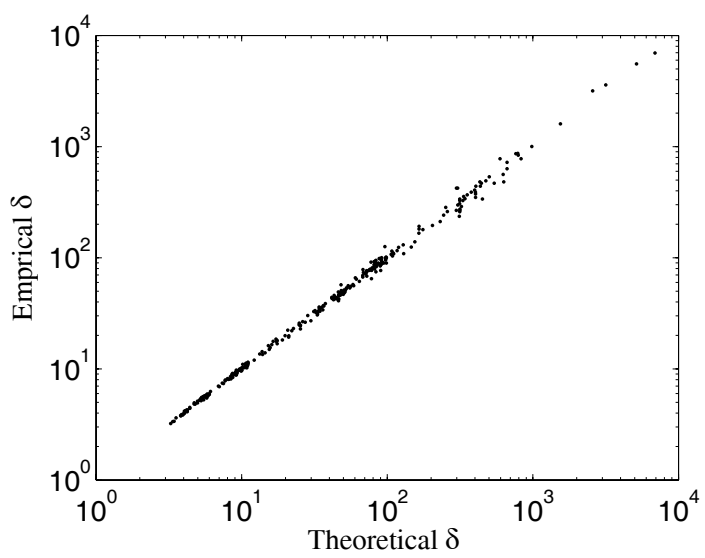

Figure 1: Comparison of the theoretical and empirical variance reduction factor for the CMC simulation with $p=0.90$.

ranging from 13 to 30 were considered, and 30 networks were randomly generated for each level of network density. In addition, experiments were repeated for three different arc reliability levels, $p=0.90,0.95$, and 0.99 . In all cases, $K=10^{6}$ was used, and $R(G)$ was calculated exactly for each network using a factoring algorithm (Page and Perry 1991), which enabled to calculate the theoretical variance reduction factor $(\delta)$ given in (11) for CMC.

Table 1 shows the minimum, median, and maximum theoretical variance reductions obtained for random networks using CMC simulation. As seen in the table, the median variance reduction factors were 31, 115, and 2566 for $p=0.90,0.95$, and 0.99, respectively. Figure 1 illustrates a comparison of the theoretical and actual variance reductions for random networks. As seen in the figure, the experimental results are highly consisted with the theoretical results. The results showed that the higher is the reliability of a network, the higher level of variance reduction obtained.

In the second part of experiments, instead of $\mathrm{CMC}$, the SC method (Easton and Wong 1980) was used to estimate allterminal reliability in order to investigate the effect of using network reductions with a variance-reduction simulation technique. Tables 2 and 3 summarize the results for the SC method. Table 2 presents the variance reductions due only to the network reductions. The results in this table were obtained as follows. First, the reliability of each network was estimated using the SC method, and the variance of this estimation was calculated from the samples taken during the simulation. Let $\operatorname{Var}_{B}$ denote the variance before reductions. Then, network reductions were applied, and reliability was 
Table 2: Results for the sequential construction method.

\begin{tabular}{cccc}
\hline & \multicolumn{3}{c}{ Var $_{B} /$ Var $_{A}$} \\
\cline { 2 - 4 }$p$ & minimum & median & maximum \\
\hline 0.90 & 4 & 185 & 89340 \\
0.95 & 10 & 1995 & 6705195 \\
0.99 & 202 & 1041845 & 1147275074272 \\
\hline
\end{tabular}

Table 3: Combined effect of network reductions and sequential construction method.

\begin{tabular}{cccc}
\hline & \multicolumn{3}{c}{ Var [] in $(5) /$ Var $_{A}$} \\
\cline { 2 - 4 }$p$ & minimum & median & maximum \\
\hline 0.90 & 18 & 449 & 193175 \\
0.95 & 83 & 7039 & 25583911 \\
0.99 & 14484 & 10361703 & 5600347736444 \\
\hline
\end{tabular}

estimated one more time using the SC method. Let $\operatorname{Var}_{A}$ be the variance of the estimation after applying reductions. The results given in Table 2 are the ratio of $\operatorname{Var}_{B}$ to $\operatorname{Var}_{A}$. Therefore, the variance reduction factors in Table 2 are independent from the variance reductions due to the SC method. As seen in the table, network reductions provided considerably higher reductions in the variance when used with the SC method than with CMC. This additional gain in variance reduction can be explained as follows. Compared to $\mathrm{CMC}$, the performance of the SC method increases with increasing network reliability (Fishman 1987, Easton and Wong 1980). As seen in Figure 3, the ratio of the variance of CMC to the variance of the SC method exponentially increases as reliability approaches one. This property of the $\mathrm{SC}$ method can be exploited in reduced networks since the reliability of a reduced network $G^{\prime}$ is always higher than that of the original network $G$. Therefore, the SC method provided an additional variance reduction.

In Table 3, the ratio of the variance of CMC given in (5) to $\left(V a r_{A}\right)$ is given. This ratio indicates the combined effect of network reductions and the SC method in reducing the sampling error. As the results show that if network reduction techniques are used along with an efficient variance reduction approach such as the SC method, very high levels of variance reductions can be achieved. Similar to $\mathrm{CMC}$, the level of variance reduction considerably increased with increasing arc reliability.

When the results were carefully analyzed, it was realized that the highest variance reductions were achieved for two cases, very sparse and very dense networks. An example for this observation is given in Figure 2 where the median variance reduction is plotted against the number of arcs. Random sparse networks usually had several nodes to apply network reductions, and as a result sparse networks were able to be greatly simplified through network reductions. This provided high levels of reductions in variance as most of the network states were covered through reductions. In dense networks, for most cases only one or two reductions could be applied, but these few reductions provided very high levels of reduction in variance. Note that $1>\lambda>R(G)$ and $R\left(G^{\prime}\right)>R(G)$ if a network $G$ is reducible through network reductions, and a dense network is usually highly reliable (i.e., $R(G) \approx 1.0$ ). Therefore, $\lambda-R(G)$ becomes very small $(\approx 0)$ in the case of a reducible dense network, and in turn results in a large reduction in the variance due to (11) for CMC. As mentioned earlier, the SC method yields exponentially smaller variance as reliability increases, and after reductions, the reliability of the reduced network becomes almost one for a highly reliable dense network. This also explains very high levels of variance reductions which were obtained for dense networks when the SC method was used.

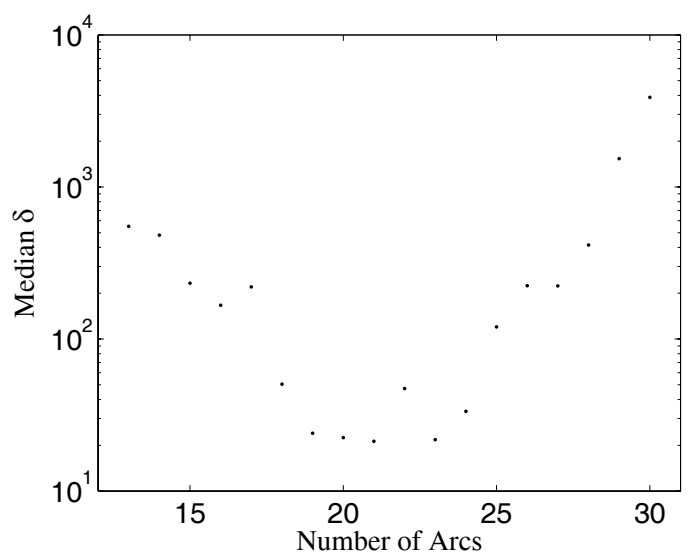

Figure 2: Median variance reduction factor versus network density for the SC Method with $p=0.90$.

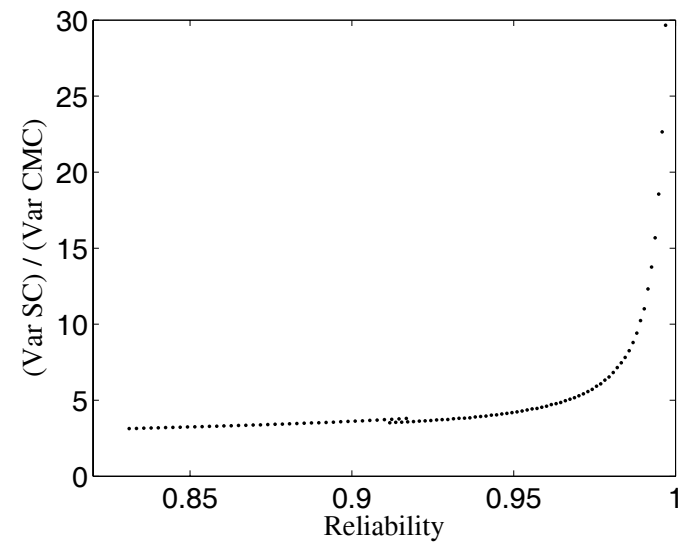

Figure 3: The ratio of the variance of the SC method to the variance of $\mathrm{CMC}$ as a function of reliability.

\section{CONCLUSIONS}

Simulation is commonly used to estimate network reliability since its exact computation is computationally intractable. As both theoretical and empirical results have shown, applying network reductions before simulation provides significant variance reduction for the estimator. The proposed 
approach is a general tool that can be easily used with other variance reduction techniques a demonstrated.

\section{REFERENCES}

Aboelfotoh, H., and C. Colbourn. 1989. Series-parallel bounds for the two-terminal reliability problem. ORSA Journal on Computing 1 (4): 209-22.

Ball, M. 1979. Computing network reliability. Operations Research 27:823-38.

Ball, M., and R. V. Slyke. 1977. Backtracking algorithms for network reliability analysis. Discrete Applied Mathematics 1:49-64.

Brecht, T., and C. Colbourn. 1988. Lower bounds on twoterminal network reliability. Discrete Applied Mathematics 21 (3): 185-98.

Cancela, H., and M. E. Khadiri. 1995. A recursive variancereduction algorithm for estimating communicationnetwork reliability. IEEE Transactions on Reliability 44 (4): 595-602.

Cancela, H., and M. E. Khadiri. 1998. A series-parallel reductions in monte carlo network-reliability. IEEE Transactions on Reliability 47 (2): 159-164.

Cancela, H., and M. E. Khadiri. 2003. The recursive variance-reduction simulation algorithm for network reliability evaluation. IEEE Transactions on Reliability 52 (2): 207-12.

Colbourn, C. 1987. Combinatorics of network reliability. New York, NY, USA: Oxford.

Deeter, D., and A. Smith. 1998. Economic design of reliable networks. IIE Transactions 30 (12): 1161-74.

Easton, M., and C. Wong. 1980. Sequential destruction method for monte carlo evaluation of system reliability. IEEE Transactions on Reliability R-29 (1): 27-32.

Elperin, T., I. Gertsbakh, and M. Lomonosov. 1991. Estimation of network reliability using graph evolution models. IEEE Transactions on Reliability 40 (5): 572-81.

Fishman, G. 1986a. A comparison of four monte carlo methods for estimating the probability of s-t connectedness. IEEE Transactions on Reliability R-35 (2): 145-55.

Fishman, G. 1986b. A monte carlo sampling plan for estimating network reliability. Operations Research 34 (4): 581-94.

Fishman, G. 1987. A monte carlo sampling plan for estimating reliability parameters and related functions. Networks 17 (2): 169-86.

Kumamoto, H., K. Tanaka, and K. Inoue. 1977. Efficient evaluation of system reliability by monte carlo method. IEEE Transactions on Reliability R-26 (5): 311-15.

Kumamoto, H., K. Tanaka, K. Inoue, and E. Henley. 1980. Dagger-sampling monte carlo for system unavailability evaluation. IEEE Transactions on Reliability R-29 (2): 122-5.
Lam, Y., and V. Li. 1986. An improved algorithm for performance analysis of networks with unreliable components. IEEE Transactions on Communications COM-34 (5): 496-7.

Li, V., and J. Silvester. 1984. Performance analysis of networks with unreliable components. IEEE Transactions on Communications COM-32 (10): 1105-10.

Lomonosov, M., and V. Polesskii. 1972. Lower bound of network reliability. Problemy Peredachi Informatsii 8 (2): 118-23.

Mazumdar, M., D. Coit, and K. McBride. 1999. A highly efficient monte carlo method for assessment of system reliability based on a markov model. American Journal of Mathematical and Management Sciences 19 (1-2): 115-33.

Page, L., and J. Perry. 1991. A note on computing environments and network reliability. Microelectronics and Reliability 31 (1): 185-6.

Resende, L. 1988. Implementation of a factoring algorithm for reliability evaluation of undirected networks. IEEE Transactions on Reliability 37 (5): 462-8.

Resende, M. 1986. A program for reliability evaluation of undirected networks via polygon-to-chain reductions. IEEE Transactions on Reliability R-35 (1): 24-9.

Satyanarayana, A., and M. Chang. 1983. Network reliability and the factoring theorem. Networks 13 (1): 107-20.

Slyke, R. V., and H. Frank. 1972. Network reliability analysis. i. Networks 1 (3): 279-90.

Srivaree-ratana, C., A. Konak, and A. Smith. 2002. Estimation of all-terminal network reliability using an artificial neural network. Computers and Operations Research 29 (7): 849-68.

Yang, C.-L., and P. Kubat. 1989. Efficient computation of most probably states for communication networks with multimode components. IEEE Transactions on Communications 37 (5): 535-8.

\section{AUTHOR BIOGRAPHY}

ABDULLAH KONAK is an Assistant Professor of Information Sciences and Technology at the Pennsylvania State University Berks. He received his B.S. degree in Industrial Engineering from Yildiz Technical University, M.S. in Industrial Engineering from Bradley University, and Ph.D. in Industrial Engineering from University of Pittsburgh. Previous to this position, he was an instructor in the Department of Systems and Industrial Engineering at Auburn University for two years. His current research interest is in the application of Operations Research techniques to complex problems, including such topics as telecommunication network design, network reliability analysis/optimization, facilities design, and data mining. He is a member of IIE and INFORMS. His web page can be found via <www.personal.psu. edu/auk3>. 